Published in final edited form as:

Prog Neuropsychopharmacol Biol Psychiatry. 2001 October ; 25(7): 1421-1433.

\title{
EFFECTS OF CHRONIC COCAINE ADMINISTRATION ON AGGRESSIVE BEHAVIOR IN VIRGIN RATS
}

\author{
DEBORAH A. LUBIN ${ }^{1}$, KATHLEEN E. METER ${ }^{3}$, CHERYL H. WALKER $^{2}$, and JOSEPHINE M. \\ JOHNS $^{2}$ \\ ${ }^{1}$ Department of Psychology, University of North Carolina at Chapel Hill, Chapel Hill, NC, USA \\ 2 Department of Psychiatry, University of North Carolina at Chapel Hill, Chapel Hill, NC, USA \\ ${ }^{3}$ Department of Psychiatry, Duke University, Durham, NC, USA
}

\section{Abstract}

1. Virgin Sprague-Dawley rats received subcutaneous injections of saline, $3.5,7.5 \mathrm{or} 15 \mathrm{mg} /$ $\mathrm{kg}$ of cocaine, twice daily, for 20 consecutive days.

2. Females were videotaped for 10 minutes in the presence of a male rat for assessment of aggression towards the intruder 2, 3, and 5 days following cessation of cocaine or saline administration. Oxytocin levels in discrete brain areas were assayed following behavioral testing, 5 days following cessation of cocaine or saline administration.

3. The $30 \mathrm{mg} / \mathrm{kg}$-dose group tended to have a lower frequency of fight attacks and aggressive postures compared to saline-treated controls across sessions.

4. The frequency of most of the behaviors analyzed were represented by quadratic functions across time, such that the highest frequency of behavior occurred 2 days following the final injection with relatively less activity 3 and 5 days following cessation of saline or cocaine administration.

5. The $30 \mathrm{mg} / \mathrm{kg}$ cocaine-treated group had significantly lower hippocampal OT levels than the $15 \mathrm{mg} / \mathrm{kg}$ group 5 days following cessation of cocaine or saline administration.

\author{
Keywords \\ amygdala; cocaine; maternal aggression; oxytocin
}

\section{Introduction}

The public perception of violence associated with cocaine abuse is typified in anecdotal reports of crime related to the procurement of the abused substance. However, increases in aggressive behavior in chronic users (Miller et al 1991;Moeller et al 1994;Yudofsky et al 1993) as well as in non-users following acute administration of cocaine (Licata et al 1993) have been reported, independent of drug acquisition. Most of the literature examining cocaine-induced alterations of aggressive behavior in humans reflects only male participants. Yet recent investigations have linked gestational cocaine use with increased levels of child abuse and neglect (Tyler et al 1997; Wasserman and Leventhal 1993). Also, 
Goldstein et al (1991) found differences in the types of violent behaviors associated with cocaine use between men and women, but data collected on violence towards children was only obtained for the female sample and thus was excluded from further analysis.

In order to avoid the many confounds associated with studies of substance abuse in humans, various animal models have been employed to study the relationship between cocaine administration and aggressive behavior. In rats, chronic gestational cocaine exposure has been correlated with subsequent increased levels of maternal aggressive behavior towards a home cage intruder (Heyser et al 1992;Johns et al 1994;Johns et al 1995; Johns et al 1997; Johns et al 1998b) and decreased levels of oxytocin (a nonapeptide critical to the initiation of maternal behavior in rats) in the amygdala (Johns et al 1995;Johns et al 1997). While several experiments have demonstrated heightened levels of maternal aggression in rats given moderately high doses of cocaine $(15 \mathrm{mg} / \mathrm{kg}$ or $20 \mathrm{mg} / \mathrm{kg}$, twice daily) throughout gestation, we are unaware of any investigations examining whether cocaine similarly increases aggressive behavior toward an intruder male, in virgin females.

Mayer et al, (1987) reported that singly housed, untreated virgin female rats were much less likely to attack a home cage intruder than lactating females. Erskine et al (1978) also reported relatively low levels of aggressive behavior toward an intruder regardless of whether the virgin females were housed in isolation for as little as 4 days or as long as 20 days. In addition, Erskine et al (1978) found no differences in the frequency of attacks relative to phase of the estrous cycle, with $89 \%$ of their virgin subjects attacking a male intruder at least once during a 10 minute testing session (Erskine et al 1978).

Ovariectomized females tested for aggressive behavior twice weekly for 7 weeks; treated in the 8 week with estrogen alone (inducing proestrus); and in the $9^{\text {th }}$ week with estrogen and progesterone (inducing behavioral estrus) maintained virtually the same levels of aggressive behavior toward a male intruder across sessions (DeBold and Miczek 1981). In the absence of ovarian steroids, non-lactating females maintained consistent levels of aggressive behavior toward an intruder before and up to 7 weeks following ovariectomy (DeBold and Miczek 1984). However, several investigators have determined that untreated lactating females are much more aggressive towards a home cage intruder than virgin females (Erskine et al 1978;Mayer et al 1987;Mayer and Rosenblatt 1993). It has been suggested that the onset of maternal aggression is under hormonal control as ovariectomized virgin females become more aggressive toward an intruder when treated with estrogen and progesterone, in a regimen paralleling late pregnancy (Mayer et al 1990). Therefore, it seems that the heightened aggressive activity displayed by lactating dams may be at least partially driven by the unique hormonal environment surrounding parturition, while aggressive behavior in virgin females may be more independent of normally cycling ovarian steroids.

The current study was conducted to determine whether chronic cocaine administration would produce similar increases in aggressive behavior (towards an intruder male) in virgin rats as previously found in lactating rats (Heyser et al 1992;Johns et al 1994;Johns et al 1997; Johns et al 1998b;Lubin et al 1998). The dynamic of behavior across test sessions was also explored. Finally, whether cocaine-induced alterations in aggressive behavior were correlated with changes in amygdaloid OT levels in virgin females was investigated.

\section{Methods}

\section{Animals}

Female Sprague-Dawley rats (200-225g) were group housed in temperature and humidity controlled rooms for a one week habituation period prior to random assignment to one of four treatment groups (see treatment). Upon assignment to a treatment group, the virgins 
were singly housed and maintained on a reversed $12 \mathrm{hr}: 12 \mathrm{hr}$ light cycle (with lights out at 0900) for 8 days, then transferred to a room with a regular light cycle (lights on at 0700) for the remainder of the experiment - consistent with the housing conditions for pregnant animals housed in the same animal quarters. All females were allowed ad libitum access to food and water except those in the saline control group which were "yoke-fed" to females in the $30 \mathrm{mg} / \mathrm{kg}$ cocaine treatment group. Yoke-fed females were offered only the amount of food eaten by a female in the high dose cocaine group on the corresponding treatment day. This procedure was employed to control for the potential anorectic effects of cocaine treatment on weight gain.

\section{Treatment}

Virgin females were assigned to one of three cocaine hydrochloride dose groups or a yokefed saline control (10 females per group). Cocaine-treated females were injected subcutaneously, on alternating flanks, twice daily (bid), with either 15,75 , or $3.75 \mathrm{mg} / \mathrm{kg}$ of cocaine dissolved in $0.9 \%$ normal saline for a total volume of $1 \mathrm{ml} / \mathrm{kg}$. Saline-treated females received $1 \mathrm{ml} / / \mathrm{kg} 0.9 \%$ normal saline, bid. Injections were given at 0800 and 1600 for 20 consecutive days. Subcutaneous injections of $30 \mathrm{mg} / \mathrm{kg}$ cocaine caused some skin lesions after about 15 days of injections but did not appear to cause the rats notable distress. As skin lesions are prone to develop following repeated subcutaneous administration of cocaine solution, injection sites were varied. Also, skin lesions that developed were cleaned with a betadine wash and a topical antibacterial ointment (Polymycin-Bacitracin-Neomycin, Burroughs Wellcome, Raleigh, N.C.) was applied as soon as they were discovered.

\section{Drug}

Cocaine was dissolved in normal (0.9\%) saline. The solutions were stored in amber bottles and maintained in a standard refrigerator when not in use. New solutions were prepared approximately every three days.

\section{Aggression Testing}

Two days following the final injection of saline or cocaine, each female was brought to the observation room in her home cage. Her weight was recorded and she was allowed to habituate to the room for 5 minutes. A smaller (175-200g) intruder male was then introduced into the home cage. The interaction between male and female was videotaped for a ten-minute period beginning as soon as the male was placed in the cage, and later scored by two independent observers, blind to treatment conditions, for 11 behaviors exhibited by the female, including:

Push/Box/Kick—Female pushes or kicks the intruder away from her body with her paws or whole body.

Receptive Behavior-Female displays ear wiggling, hop darting and/or lordosis.

Aggressive Posture-Female stands over the intruder, which is usually lying on it's back, and tries to force it into a full submissive posture by pushing with front paws.

Fight Attack-A quick lunge by the female usually followed by rolling, biting and fur pulling directed towards the neck and back regions.

Selfgroom-Female grooms herself with her tongue or forepaws. 
Lateral/Front Threat-Female turns her flank towards intruder with her back arched and legs extended, moving laterally and usually pushing against intruder. Front threat involves dam extending her nose and neck stiffly towards the intruder sometimes with teeth chattering. Intruder responds by freezing or escaping.

Defensive/Submissive Posture-Female lies on her side or back with her ventral surface exposed, usually immobile with legs extended.

Rough Groom/Nip—Female nips or grooms intruder male roughly.

Locomotion-Female is ambulatory and moves all four paws in one direction.

Rear/Sniff-Female rears on hind legs and sniffs the top or sides or rear of cage.

Other-Any other behavior other than those included in the categories above.

Test sessions were immediately discontinued if the female mortally wounded the intruder, and data from that session were not included in the statistical analysis. Behavior was independently scored by two individuals for frequency, duration and latency using a computer program specifically designed for that purpose. Inter-rater agreement was within $90 \%$ (or better) for frequency and latency and within $80 \%$ (or better) for duration of behavior. Following the testing procedures, the animals were returned to the colony room. Identical procedures were followed 3 days and 5 days following the cessation of cocaine or saline administration.

\section{Brain Dissections}

Immediately following the final aggression testing session (5 days following the last injection), females were decapitated and the whole hippocampus, amygdala, and ventral tegmental area (VTA), of their brains were dissected on ice, weighed, and rapidly frozen and stored at $-70 \mathrm{C}$ for subsequent oxytocin radioimmunoassay. Briefly, brains were coronally sectioned from the ventral side rostral to the optic chiasm [approximately A7100 according to Konig and Klippel (1963)] and just caudal to the optic chiasm (approximately A5800) to define the preoptic-anterior hypothalamic area. The brains were sectioned once again just caudal to the tuber cinereum (approximately A3800) to define the medial basal hypothalamus. The amygdala was removed from these two sections. The whole hippocampus was then removed from the caudal remainder of the brain, and the ventral tegmental area dissected from this portion by making dorso-ventral cuts medial to the optic tracts with a dorsal cut at the ventral extent of the central gray.

\section{Oxytocin (OXY) Radioimmunoassay}

Brain tissue samples from the hippocampus, amygdala and VTA were homogenized in 1.5, 0.6 , or $0.5 \mathrm{ml}$, respectively, of cold buffer ( $19 \mathrm{mM}$ monobasic sodium phosphate, $81 \mathrm{mM}$ dibasic sodium phosphate, $0.05 \mathrm{M}$ sodium chloride, $0.1 \%$ Bovine albumin serum, $0.1 \%$ triton $\mathrm{X}-100$, and $0.01 \%$ sodium azide at a $\mathrm{pH}$ of 7.4 ). Samples were then centrifuged at $3000 \mathrm{x} \mathrm{g}$ for $30 \mathrm{~min}$. The subsequent supernatants were assayed for oxytocin immunoreactive content using a protocol and reagents from Peninsula Laboratories Inc. (Belmont, California).

Briefly, oxytocin-like immunoreactivity was assayed by incubating samples and standards $(0.5-500 \mathrm{pg})$ in duplicate, at $0 \mathrm{C}$, for 16-24 hours with rabbit anti-oxytocin serum. The tracer, ${ }^{125} \mathrm{I}$-Oxytocin (specific activity $=1286 \mathrm{Ci} / \mathrm{mmol}$ ), was incubated with samples and standards for $16-24$ hours at $0 \mathrm{C}$. Then, the ${ }^{125} \mathrm{I}$-Oxytocin bound to the antibody was separated from free by adding $0.1 \mathrm{ml}$ normal rabbit serum and $0.1 \mathrm{ml}$ goat anti-rabbit $\operatorname{IgG}$ and incubating for 2 hours, at room temperature. Then $500 \mathrm{ml}$ of buffer were added and the 
tubes were centrifuged at $2500 \times \mathrm{g}$ for 40 minutes. Radioactivity in the pellet was measured by an LKB Clinigamma counter which estimates $\mathrm{B} / \mathrm{B}_{0}$ from the standard curve and estimates pg amounts from that determination for each sample. Brain concentrations were expressed as pg OXY/mg tissue Cross-reactivity with arginine vasopressin and somatostatin was undetectable. The sensitivity of the assay was $0.5 \mathrm{pg}$ oxytocin/tube and the intra-assay coefficient of variance of $<5 \%$ and an interassay coefficient of $<14 \%$ at approximately $50 \%$ binding.

\section{Statistical Analysis}

Behavioral differences were examined using a 2-way (group by test session) Analysis of Variance (ANOVA) using a general linear models approach to repeated measures analysis allowing for inclusion of individuals with missing data (e.g data from an animal 2 and 3 days following the final injection was included in the analyses whether or not the data from the $5^{\text {th }}$ day following the last injection was missing). Follow-up contrasts were conducted for significance. Oxytocin levels (pg/mg wet brain tissue weight) were statistically analyzed by ANOVA followed by Tukey's HSD post hoc analysis.

\section{Results \\ Food Consumption}

The $30 \mathrm{mg} / \mathrm{kg}$ cocaine-treated group consumed significantly more rat chow on average than the yoke-fed saline-treated controls, $F(1,18)=8.16, \mathrm{p}<.01$, although the yoke-fed females tended to gain slightly more weight over the 20 day interval (non-significant).

\section{Behavior}

There were very few dose-dependent differences in aggressive behavior among virgin females toward an intruder male. While there was a significant main effect of group in the frequency of fight attacks $\mathrm{F}(3,36)=3.89, \mathrm{p}<.02$, and aggressive postures, $\mathrm{F}(3,36)=4.16$, $\mathrm{p}<$. 01, follow-up contrasts reveal group differences that were not statistically significant (except a single group difference in aggressive posture) (Fig. 1).

A repeated measures ANOVA indicated that there was a systematic variation in behavior within groups, and in some cases between groups, across time. That is, in each of the metrics recorded (frequency, duration, latency), 8 behaviors were represented by quadratic functions The highest frequencies of receptive behavior, rough groom/nip, selfgroom, fight attack, defensive behavior, and aggressive posture were observed 2 days after the final injection of saline or cocaine and were generally followed by a precipitous drop 3 and 5 days following the final injection. Alternatively, there was a lower frequency of rear/sniff and "other" behaviors 2 days following chronic injections with an increase in these behaviors on the $3^{\text {rd }}$ and $5^{\text {th }}$ day following the final injection. These asymptotic functions were typified by significant rates of change of behavior in each of the groups with few significant differences in slope among groups (Table 1).

There were also significant interactions of group and session in terms of duration and latency of the behaviors examined. The $30 \mathrm{mg} / \mathrm{kg}$ group consistently had a significantly smaller rate of change (in duration of fight attack, rear/sniff and "other" behavior, and in latency to push/box/kick, exhibit receptive behavior, and rear/sniff) across the testing period relative to the other groups (data not shown).

\section{Oxytocin Levels}

Five days following cessation of cocaine or saline administration, there were no significant differences in OT levels in the amygdalas or VTAs of the virgin females. There was a 
significant group difference in OT levels in the hippocampus, $F(3,36)=4.07, p<.01$. The 30 $\mathrm{mg} / \mathrm{kg}$ cocaine-treated dams had significantly lower hippocampal OT levels compared to the $15 \mathrm{mg} / \mathrm{kg}$ cocaine-treated group, $\mathrm{p}<.02$.

\section{Discussion}

\section{Relationship To Previous Investigations}

Based on cocaine-induced increases in maternal aggressive behavior in lactating dams (Heyser et al 1992;Johns et al 1994;Johns et al 1997;Johns et al 1998b), and studies of aggression in virgin rats (DeBold and Miczek 1981; DeBold and Miczek 1984; Erskine et al 1978; Mayer and Rosenblatt 1993), it was hypothesized that chronic cocaine administrations would either produce dose-related increases in aggression or negligible behavioral effects in virgin females. Therefore, the dose dependent trend toward decreased frequencies of fight attack and aggressive postures in the $30 \mathrm{mg} / \mathrm{kg}$ virgin group was unanticipated. The relatively low levels of aggressive behavior in this group were not attributable to increases in stereotypical, hyperactive behaviors. Whether withdrawal from cocaine played a role in altered aggressive behavior is unknown, however no animal exhibited overt signs of withdrawal or appeared ill. Previous investigations have documented that lactating dams either withdrawn from cocaine or maintained on a twice daily regimen during the postpartum period exhibited equivalent increases in aggressive behavior (Johns et al 1997) Further experiments are required to determine the potential role of withdrawal on aggressive behavior in virgin females.

Two important observations distinguish the behavior of the virgins in this investigation from that of parturient dams in previous work. First, in chronically treated parturient dams, the $30-40 \mathrm{mg} / \mathrm{kg}$ group typically exhibited the highest levels of aggressive behavior (Heyser et al 1992;Johns et al 1994;Johns et al 1997; Johns et al 1998b). In virgin rats, the $30 \mathrm{mg} / \mathrm{kg}$ group tended to exhibit the lowest frequencies of aggressive behavior. The virgin pattern of behavior more closely parallels that of untreated, parturient dams given acute cocaine injections during the postpartum period (Johns et al 1998a). Perhaps the sharp increase in oxytocinergic activity in the peripartum period (Insel 1986) and its modification by chronic cocaine administration is necessary for the induction of increases in aggressive behavior.

Second, parturient dams given cocaine or saline throughout gestation (20 days) and tested for maternal aggression on postpartum days 2, 3 and 5, maintained relatively consistent frequencies of 11 behaviors across test sessions (Lubin et al 1998). The virgin females, on the other hand, had a much higher frequency of almost all evaluated behaviors 2 days relative to 3 and 5 day following cessation of cocaine or saline administration (although the $30 \mathrm{mg} / \mathrm{kg}$ treated group tended to have more consistent levels of aggressive behavior across sessions compared to saline-treated virgins). As increased activity on the $2^{\text {nd }}$ injection free day was reflected in both cocaine and saline-treated animals, attributing this phenomenon to some type of withdrawal syndrome is premature. Further investigations including evaluations of behavior 1 day following the final cocaine or saline injection should be conducted.

\section{Possible Mechanisms}

Disparate levels of cocaine-induced aggression between virgins and lactating animals may be attributed to many factors including differential pharmacokinetic profiles of cocaine in pregnant versus non-pregnant females. While Duhart et al (1993) demonstrated that the pharmacokinetics of cocaine and benzoylecgonine (a cocaine metabolite) were very similar between chronically treated pregnant versus acutely treated non-pregnant Rhesus Monkeys, Church and Subramanian (1997) demonstrated increased benzoylecgonine levels and 
increased mortality in rats during late versus mid gestation. Also, Vernotica and Morrell (1998) found that while acute injections of cocaine did not yield statistically significantly different plasma cocaine levels, the lactating females consistently had higher mean plasma cocaine levels than virgins (Vernotica and Morrell 1998). Therefore, differential levels of absorption, distribution as well as altered levels of active and inactive metabolites may have affected behavioral outcomes (Morishima and Whittington 1995). Finally, in this investigation, normally cycling virgin rats were used. We did not allow sensitization to pups, which has been shown to increase maternal aggressive behavior (Mayer and Rosenblatt 1993). Instead, the interest was in determining whether chronic cocaine-induced enhancement of aggressive behavior and correlated decreases in OT levels were unique to the lactating female or might also be manifest in virgin females

Perhaps importantly, the $30 \mathrm{mg} / \mathrm{kg}$-treated virgin group had significantly less OT in their hippocampi, relative to the other virgin groups. Unlike the relationship of decreased OT levels in the amygdala to increases in aggressive behavior in lactating dams, the role of decreased OT levels in the hippocampus has not been established in lactating or virgin rats Further investigation is required to determine whether altered OT system dynamics facilitate alterations in aggressive behavior in cocaine-treated virgins.

\section{Conclusion}

The hormonal milieu of pregnancy and lactation seems to produce more robust increases in aggressive behavior than normally cycling ovarian steroids, and chronic cocaine administration may exaggerate that disparity. Minimal alterations in aggression were apparent in virgin rats 23 , and 5 days following the cessation of cocaine or saline administration. Therefore, future investigations will explore potential behavioral and biochemical differences between withdrawn and non-withdrawn virgin groups (1 day following cessation of chronic cocaine administrations) and include evaluations of aggressive behavior corresponding to a time when significant increases in maternal aggression have been found in lactating animals (6 days following final injections). Also parallel investigations of the effects of chronic cocaine administration on aggressive behavior in castrated versus intact male rats would be an important addition to the literature.

\section{Acknowledgments}

This research was supported by NIH grant DA08456 awarded to JMJ.

\section{Abbreviations}

$\begin{array}{ll}\text { AMY } & \text { amygdala } \\ \text { PPD } & \text { postpartum day } \\ \text { OT } & \text { oxytocin } \\ \text { VTA } & \text { ventral tegmental area }\end{array}$

\section{References}

CHURCH MW, SUBRAMANIAN MG. Cocaine's Lethality increases during late gestation in the rat. Am J Obstet Gynecol. 1997; 176:901-906. [PubMed: 9125619]

DEBOLD JF, MICZEK KA. Sexual Dimorphism in the Hormonal Control of Aggressive Behavior of Rats. Pharm Biochem Behav. 1981; 14:89-93.

DEBOLD JF, MICZEK KA. Aggression Persists After Ovariectomy in Female Rats. Horm Behav. 1984; 18:177-190. [PubMed: 6539749] 
DUHART HM, FOGLE CM, GILLAM MP, BAILEY JR, SLIKKER WJR, PAULE MG. Pharmacokinetics of Cocaine In Pregnant and Nonpregnant Rhesus Monkeys. Reprod Toxicol. 1993; 7:429-437. [PubMed: 8274818]

ERSKINE MS, BARFIELD RJ, GOLDMAN BD. Intraspecific Fighting During Late Pregnancy and Lactation in Rats and Effects of Litter Removal Behav. Biol. 1978; 23:206-218.

GOLDSTEIN PJ, BELLUCCI PA, SPUNT BJ, MILLER T. Frequency of cocaine use and violence: a comparison between men and women. NIDA Res Monogr. 1991; 110:113-138. [PubMed: 1944494]

HEYSER CJ, MOLINA VA, SPEAR LP. A fostering study of the effects of prenatal cocaine exposure: I. Maternal behaviors. Neurotoxicol Teratol. 1992; 14:415-421. [PubMed: 1488036]

INSEL TR. Postpartum increases in brain oxytocin binding. Neuroendocrinol. 1986; 44:515-518.

JOHNS JM, FAGGIN BM, NOONAN LR, LI L, ZIMMERMAN LI, PEDERSEN CA. Chronic cocaine treatment decreases oxytocin levels in the amygdala and increases maternal aggression in Sprague-Dawley rats. Society for Neurosci Abstracts. 1995; 21:766.7.

JOHNS JM, NELSON CJ, METER KE, LUBIN DA, COUCH CD, AYERS A, WALKER CH. DoseDependent Effects of Multiple Acute Cocaine Injections on Maternal Behavior and Aggression in Sprague-Dawley Rats. Dev Neurosci. 1998a; 20:525-532. [PubMed: 9858841]

JOHNS JM, NOONAN LR, ZIMMERMAN LI, LI L, PEDERSEN CA. Effects of chronic and acute cocaine on the onset of maternal behavior and aggression in Sprague-Dawley rats. Behav Neurosci. 1994; 108:107-112. [PubMed: 8192835]

JOHNS JM, NOONAN LR, ZIMMERMAN LI, LI L, PEDERSEN CA. Effects of short- and longterm withdrawal from gestational cocaine treatment on maternal behavior and aggression in Sprague-Dawley rats. Dev Neurosci. 1997; 19:368-374. [PubMed: 9215883]

JOHNS, JM.; NOONAN, LR.; ZIMMERMAN, LI.; McMILLEN, BA.; MEANS, LW.; WALKER, CH.; LUBIN DAMETER, KE.; NELSON, CJ.; PEDERSEN, CA.; MASON, GA.; LAUDER, JM. Chronic Cocaine Treatment Alters Social/Aggressive Behavior in Sprague-Dawley Rat Dams and in Their Prenatally Exposed Offspring. In: Harvey, JA.; Kosofsky, B., editors. Cocaine: Effects on the Developing Brain. The New York Academy of Sciences; New York: 1998b. p. 399-404.

KINSLEY CH, TURCO D, BAUER A, BEVERLY M, WELLMAN J, GRAHAM AL. Cocaine alters the onset and maintenance of maternal behavior in lactating rats. Pharm Biochem Behav. 1994; 47:857-864.

KONIG, JFR.; KLIPPEL, RA. The Rat Brain: A Stereotaxic Atlas of the Forebrain and Lower Parts of the Brain Stem. Krieger; New York: 1963.

LICATA A, TAYLOR S, BERMAN M, CRANSTON J. Effects of Cocaine on Human Aggression. Pharm Biochem Behav. 1993; 45:549-552.

LUBIN DA, METER KE, WALKER CH, MURTY L, JOHNS JM. Dose-Related Effects of Chronic Gestational Cocaine Treatment on Maternal Aggression in Sprague-Dawley Rats on Postpartum Days 2, 3, and 5. Society for Neuroscience Abstracts. 1998; 24:780.13.

MAYER AD, MONROY MA, ROSENBLATT JS. Prolonged estrogen-progesterone treatment of nonpregnant ovariectomized rats: Factors stimulating home-cage and maternal aggression and short-latency maternal behavior. Horm Behav. 1990; 24:342-364. [PubMed: 2227848]

MAYER AD, REISBICK S, SIEGEL HI, ROSENBLATT JS. Maternal Aggression in Rats: Changes Over Pregnancy and Lactation in a Sprague-Dawley Strain. Aggress Behav. 1987; 13:29-43.

MAYER AD, ROSENBLATT JS. Persistent Effects on Maternal Aggression of Pregnancy but not of Estrogen/Progesterone Treatment of Nonpregnant Ovariectomized Rats Revealed When Initiation of Maternal Behavior is Delayed. Horm Behav. 1993; 27:132-155. [PubMed: 8440514]

MILLER NS, GOLD MS, MAHLER JC. Violent Behaviors Associated with Cocaine Use: Possible Pharmacological Mechanisms. The Internat J Addict. 1991; 26:1077-1088.

MOELLER FG, STEINBERG JL, PETTY F, FULTON M, CHEREK DR, KRAMER G, GARVER DL. Serotonin and impulsive/aggressive behavior in cocaine-dependent subjects. Prog, in Neuropsychopharmacol Biol Psychiat. 1994; 18:1027-1035.

MORISHIMA HO, WHITTTNGTON RA. Species-, gender-, and pregnancy-related differences in the pharmacokinetics and pharmacodynamics of cocaine. NIDA Res Monogr. 1995; 158:2-21. [PubMed: 8594485] 
TYLER R, HOWARD J, ESPINOSA M, DOAKES SS. Placement with substance-abusing mothers vs. placement with other relatives- Infant outcomes. Child Abuse Negl. 1997; 21:337-349. [PubMed: 9134263]

VAN WIMERSMA GREIDANUS TB, MAIGRET C. The role of limbic vasopressin and oxytocin in social recognition. Brain Res. 1996; 713:153-159. [PubMed: 8724986]

VERNOTICA EM, LISCIOTTO CA, ROSENBLATT JS,,, MORRELL JI. Cocaine Transiently Impairs Maternal Behavior in the Rat. Behav Neurosci. 1996; 110:315-323. [PubMed: 8731058]

VERNOTICA EM, MORRELL JI. Plasma Cocaine Levels and Locomotor Activity after Systemic Injection in Virgin and in Lactating Maternal Female Rats. Physiol \& Behav. 1998; 64:399-407. [PubMed: 9748111]

WASSERMAN DR, LEVENTHAL JM. Maltreatment of children born to cocaine-dependent mothers. Am J Dis Child. 1993; 147:1324-1328. [PubMed: 8249955]

YUDOFSKY SC, SILVER JM, HALES RE. Cocaine and Aggressive Behavior: Neurobiological and Clinical Perspectives. Bull Menninger Clin. 1993; 57:218-226. [PubMed: 8099516] 


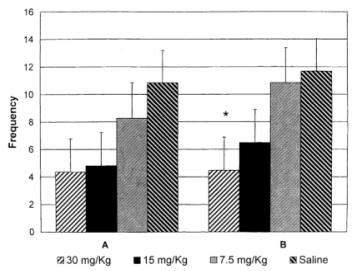

Fig 1.

Frequency of Fight Attack and Aggressive Posture. A. The saline-treated group had nonsignificantly higher rates of fighting compared to each of the cocaine-treated groups. B.

Saline-treated controls pinned intruders using an aggressive posture more often than the 30 $\mathrm{mg} / \mathrm{kg}$ cocaine-treated group $\left({ }^{*} \mathrm{p}<.04\right)$. 


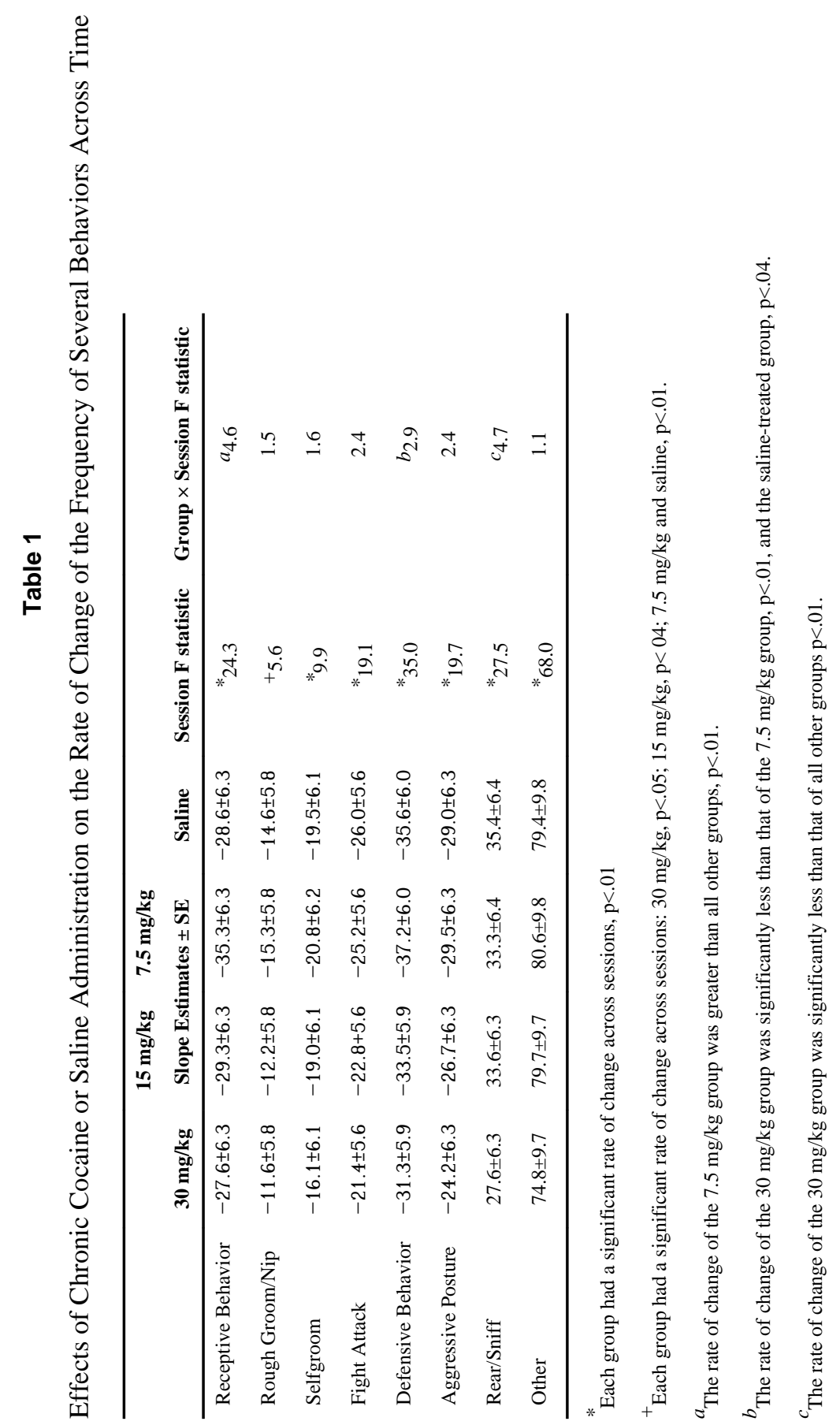

Prog Neuropsychopharmacol Biol Psychiatry. Author manuscript; available in PMC 2011 June 22. 